\title{
The Samuelson macroeconomic model as a singular linear matrix difference equation
}

\author{
Fernando Ortega and Maria Filomena Barros
}

*Correspondence:

barrosmariafilomena@gmail. com

Universitat Autonoma de

Barcelona, Bellaterra, Spain

\begin{abstract}
In this paper, we revisit the famous classical Samuelson's multiplier-accelerator model for national economy. We reform this model into a singular discrete time system and study its solutions. The advantage of this study gives a better understanding of the structure of the model and more deep and elegant results.
\end{abstract}

Keywords: Samuelson, Macroeconomic, Singular, System, Difference equations

\section{Introduction}

Many authors have studied generalized discrete, see (Apostolopoulos and Ortega 2019; Dassios 2015a; Dassios and Kalogeropoulos 2013; Ogata 1987; Ortega and Apostolopoulos 2018), and continuous time systems, see (Dassios et al. 2019, 2020; Lewis 1986, 1987, 1992; Liu et al. 2019; Milano and Dassios 2016, 2017), and their applications especially in cases where the memory effect is needed including generalized discrete, see (Dassios and Baleanu 2015; Dassios and Kalogeropoulos 2014; Dassios and Szajowski 2016), and continuous time systems with delays, see (Liu et al. 2017, 2019, 2020; Tzounas et al. 2020). Many of these results have already been extended to systems of differential, see (Dassios and Baleanu (2018a, b; Klamka 2010; Klamka and Wyrwał 2008; Podlubny 1999) and difference equations with fractional operators, see (Dassios 2015c, 2018b).

Keynesian macroeconomics inspired the seminal work of (Samuelson 1939), who actually introduced the business cycle theory. Although primitive and using only the demand point of view, the Samuelson's prospect still provides an excellent insight into the problem and justification of business cycles appearing in national economies. In the past decades, many more sophisticated models have been proposed by other researchers, see (Barros and Ortega 2019; Dassios and Devine 2016; Dassios and Zimbidis 2014; Dassios et al. 2014; Dorf 1983; Kuo 1996; Puu et al. 2004; Rosser 2000). All these models use superior and more delicate mechanisms involving monetary aspects, inventory issues, business expectation, borrowing constraints, welfare gains and multi-country consumption correlations.

Some of the previous articles also contribute to the discussion for the inadequacies of Samuelson's model. The basic shortcoming of the original model is the incapability to produce a stable path for the national income when realistic values for the different 
parameters (multiplier and accelerator parameters) are entered into the system of equations. Of course, this statement contradicts with the empirical evidence which supports temporary or long-lasting business cycles.

In this article, we propose an alternative view of the model by reforming it into a singular discrete time system.

The paper theory of difference equations is organized as follows. Section 2 provides a short review for the organization of the original model and in Sect. 3, we introduce the proposed reformulation into a system of difference equations. Section 4 investigates the solutions of the proposed system.

\section{The original model}

The original version of Samuelson's multiplier-accelerator original model is based on the following assumptions:

Assumption 2.1 National income $T_{k}$ in year $k$ equals to the summation of three elements: consumption, $C_{k}$, private investment, $I_{k}$, and governmental expenditure $G_{k}$

$$
T_{k}=C_{k}+I_{k}+G_{k} \text {. }
$$

Assumption 2.2 Consumption $C_{k}$ in year $k$ depends on past income (only on last year's value) and on marginal tendency to consume, modeled with $a$, the multiplier parameter, where $0<a<1$,

$$
C_{k}=a T_{k-1} .
$$

Assumption 2.3 Private investment $I_{k}$ in year $k$ depends on consumption changes and on the accelerator factor $b$, where $b>0$. Consequently, $I_{k}$ depends on national income changes,

$$
I_{k}=b\left(C_{k}-C_{k-1}\right)=a b\left(T_{k-1}-T_{k-2}\right) .
$$

Assumption 2.4 Governmental expenditure $G_{k}$ in year $k$ remains constant

$$
G_{k}=\bar{G} \text {. }
$$

Hence, the national income is determined via the following second-order linear difference equation

$$
T_{k+2}-a(1+b) T_{k+1}+a b T_{k}=\bar{G} .
$$

See (Samuelson 1939) for the needed theory of difference equations that lead to the solution of the above equation.

\section{The reformulation: Singular Samuelson's model}

Let

$$
Y_{k}=\left[\begin{array}{c}
T_{k} \\
C_{k} \\
I_{k}
\end{array}\right] .
$$


Then, (1) can be written as

$$
0=-T_{k}+C_{k}+I_{k}+G_{k},
$$

or, equivalently,

$$
\left[\begin{array}{lll}
0 & 0 & 0
\end{array}\right] Y_{k+1}=\left[\begin{array}{lll}
-1 & 1 & 1
\end{array}\right] Y_{k}+G_{k} .
$$

Equation (2) can be written as

$$
C_{k+1}=a T_{k}
$$

or, equivalently,

$$
\left[\begin{array}{lll}
0 & 1 & 0
\end{array}\right] Y_{k+1}=\left[\begin{array}{lll}
a & 0 & 0
\end{array}\right] Y_{k} .
$$

Finally (3) can be written as

$$
I_{k+1}=b\left(C_{k+1}-C_{k}\right) .
$$

or, equivalently,

$$
-b C_{k+1}+I_{k+1}=-b C_{k} .
$$

or, equivalently,

$$
\left[\begin{array}{lll}
0 & -b & 1
\end{array}\right] Y_{k+1}=\left[\begin{array}{lll}
0 & -b & 0
\end{array}\right] Y_{k} .
$$

Hence the above expressions can be written in a matrix form. We proved the following theorem:

Theorem 3.1 The difference equation (1) can be written in the form of the following singular discrete time system:

$$
F Y_{k+1}=G Y_{k}+V_{k}, \quad k=2,3, \ldots
$$

where

$$
F=\left[\begin{array}{ccc}
0 & 0 & 0 \\
0 & 1 & 0 \\
0 & -b & 1
\end{array}\right], \quad G=\left[\begin{array}{ccc}
-1 & 1 & 1 \\
a & 0 & 0 \\
0 & -b & 0
\end{array}\right], \quad V_{k}=\left[\begin{array}{c}
G_{k} \\
0 \\
0
\end{array}\right]
$$

Note that $F$ is singular (detF $=0$ ). Throughout the paper, we will use in several parts matrix pencil theory to establish our results. A matrix pencil is a family of matrices $s F-G$, parametrized by a complex number s, see (Dassios and Baleanu 2013).

Definition 3.1 Given $F, G \in \mathbb{R}^{r \times m}$ and an arbitrary $s \in \mathbb{C}$, the matrix pencil $s F-G$ is called

1. Regular when $r=m$ and $\operatorname{det}(s F-G) \neq 0$;

2. Singular when $r \neq m$ or $r=m$ and $\operatorname{det}(s F-G) \equiv 0$. 
Corollary 3.1 The system (4) has always a regular pencil $\forall a, b$.

Proof The determinant $\operatorname{det}(s F-G)=s^{2}-a(b+1) s+a b \neq 0$. Hence from Definition 2.1, the pencil is regular. The proof is completed.

The class of $s F-G$ is characterized by a uniquely defined element, known as the Weierstrass canonical form, see (Dassios 2017), specified by the complete set of invariants of $s F-G$. This is the set of elementary divisors of type $\left(s-a_{j}\right)^{p_{j}}$, called finite elementary divisors, where $a_{j}$ is a finite eigenvalue of algebraic multiplicity $p_{j}(1 \leq j \leq v)$, and the set of elementary divisors of type $\hat{s}^{q}=\frac{1}{s^{q}}$, called infinite elementary divisors, where $q$ is the algebraic multiplicity of the infinite eigenvalue. $\sum_{j=1}^{v} p_{j}=p$ and $p+q=m$.

From the regularity of $s F-G$, there exist non-singular matrices $P, Q \in \mathbb{R}^{m \times m}$ such that

$$
\begin{aligned}
P F Q & =\left[\begin{array}{cc}
I_{p} & 0_{p, q} \\
0_{q, p} & H_{q}
\end{array}\right], \\
P G Q & =\left[\begin{array}{cc}
J_{p} & 0_{p, q} \\
0_{q, p} & I_{q}
\end{array}\right] .
\end{aligned}
$$

$J_{p}, H_{q}$ are appropriate matrices with $H_{q}$ a nilpotent matrix with index $q_{*}, J_{p}$ a Jordan matrix and $p+q=m$. With $0_{q, p}$ we denote the zero matrix of $q \times p$. The matrix $Q$ can be written as

$$
Q=\left[Q_{p} Q_{q}\right] .
$$

$Q_{p} \in \mathbb{R}^{m \times p}$ and $Q_{q} \in \mathbb{R}^{m \times q}$. The matrix $P$ can be written as

$$
P=\left[\begin{array}{l}
P_{1} \\
P_{2}
\end{array}\right]
$$

$P_{1} \in \mathbb{R}^{p \times r}$ and $P_{2} \in \mathbb{R}^{q \times r}$.

The solution of system (4) is given by the following Theorem:

Theorem 3.2 (See Dassios 2012) We consider the system (4). Since its pencil is always regular, its solution exists and for $k \geq 0$, is given by the formula

$$
Y_{k}=Q_{p} J_{p}^{k} C+Q D_{k}
$$

Here, $D_{k}=\left[\begin{array}{c}\sum_{i=0}^{k-1} J_{p}^{k-i-1} P_{1} V_{i} \\ \operatorname{sum}_{i=0}^{q_{*}-1} H_{q}^{i} P_{2} V_{k+i}\end{array}\right]$ and $C \in \mathbb{R}^{p}$ is a constant vector. The matrices $Q_{p}, Q_{q}$, $P_{1}, P_{2}, J_{p}, H_{q}$ are defined by (5), (6) and (7).

\section{Results and discussion}

In this section we will present our main results. We will provide the solution to the system (4) and consequently we will derive the sequence for the national income, the consumption and the private investment. 
Theorem 4.1 We consider the system (4). Then in year $k$, national income $T_{k}$, consumption $C_{k}$ and private investment $I_{k}$ are given by

$$
\begin{aligned}
& T_{k}=s_{1}^{k+1} c_{1}+s_{2}^{k+1} c_{2}+a \sum_{i=0}^{k-1}\left[\left(s_{1}^{k-1}+s_{2}^{k-1}\right)\right] G_{i}, \\
& C_{k}=a\left(s_{1}^{k} c_{1}+s_{2}^{k} c_{2}\right)+a^{2} \sum_{i=0}^{k-1}\left[\left(s_{1}^{k-i-1}+s_{2}^{k-i-1}\right)\right] G_{i}, \\
& I_{k}=s_{1}^{k}\left(s_{1}-a\right) c_{1}+s_{2}^{k}\left(s_{2}-a\right) c_{2}+a \sum_{i=0}^{k-1}\left[\left(\left(s_{1}-a\right) s_{1}^{k-1}+\left(s_{2}-a\right) s_{2}^{k-1}\right)\right] G_{i} .
\end{aligned}
$$

Proof From Corollary 3.1, the pencil $s F-G$ is always regular. Furthermore, the pencil has one infinite eigenvalue and two finite:

$$
s_{1}=\frac{a(1+b)+\sqrt{a^{2}(1+b)^{2}-4 a b}}{2}, \quad s_{2}=\frac{a(1+b)-\sqrt{a^{2}(1+b)^{2}-4 a b}}{2} .
$$

From Theorem 3.2, the solution of (4) is given by

$$
Y_{k}=Q_{p} J_{p}^{k} C+Q\left[\begin{array}{c}
\sum_{i=0}^{k-1} J_{p}^{k-i-1} P_{1} V_{i} \\
\operatorname{sum}_{i=0}^{q_{*}-1} H_{q}^{i} P_{2} V_{k+i}
\end{array}\right] .
$$

Since we have one infinite eigenvalue, we have

$$
H_{q}=0
$$

and $J_{p}$ is the Jordan matrix of the two finite eigenvalues:

$$
Y_{k}=Q_{p}\left[\begin{array}{cc}
s_{1}^{k} & 0 \\
0 & s_{2}^{k}
\end{array}\right] C+Q\left[\begin{array}{c}
\sum_{i=0}^{k-1} J_{p}^{k-i-1} P_{1} V_{i} \\
0
\end{array}\right] .
$$

The matrix $Q_{p}$ has the two eigenvectors of the two finite eigenvalues:

$$
Q_{p}=\left[\begin{array}{cc}
s_{1} & s_{2} \\
a & a \\
s_{1}-a & s_{2}-a
\end{array}\right],
$$

while $Q_{q}$ is the eigenvector of the infinite eigenvalue:

$$
Q_{q}=\left[\begin{array}{l}
1 \\
0 \\
0
\end{array}\right]
$$

Hence,

$$
Q=\left[\begin{array}{ccc}
s_{1} & s_{2} & 1 \\
a & a & 0 \\
s_{1}-a & s_{2}-a & 0
\end{array}\right]
$$

and the solution $Y_{k}$ takes the form: 


$$
\begin{aligned}
Y_{k}= & {\left[\begin{array}{cc}
s_{1} & s_{2} \\
a & a \\
s_{1}-a & s_{2}-a
\end{array}\right]\left[\begin{array}{cc}
s_{1}^{k} & 0 \\
0 & s_{2}^{k}
\end{array}\right] C } \\
& +\left[\begin{array}{ccc}
s_{1} & s_{2} & 1 \\
a & a & 0 \\
s_{1}-a & s_{2}-a & 0
\end{array}\right]\left[\sum_{i=0}^{k-1}\left[\begin{array}{cc}
s_{1}^{k-i-1} & 0 \\
0 & s_{2}^{k-i-1}
\end{array}\right] P_{1} V_{i}\right] .
\end{aligned}
$$

Finally, here $P_{1}$ is the matrix which contains the right eigenvectors of the finite eigenvalues

$$
P_{1}=\left[\begin{array}{lll}
a & 1 & \frac{a}{s_{1}} \\
a & 1 & \frac{d}{s_{2}}
\end{array}\right]
$$

Hence,

$$
\begin{aligned}
& Y_{k}=\left[\begin{array}{cc}
s_{1} & s_{2} \\
a & a \\
s_{1}-a & s_{2}-a
\end{array}\right]\left[\begin{array}{cc}
s_{1}^{k} & 0 \\
0 & s_{2}^{k}
\end{array}\right] C
\end{aligned}
$$

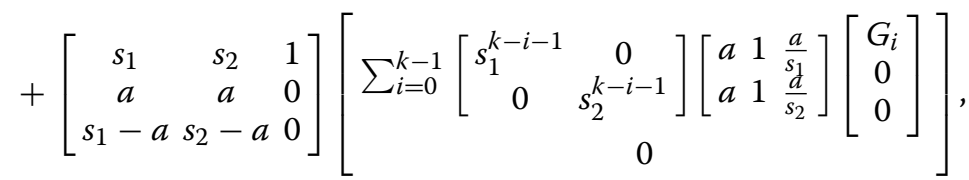

or, equivalently,

$$
Y_{k}=\left[\begin{array}{l}
s_{1}^{k+1} c_{1}+s_{2}^{k+1} c_{2}+a \sum_{i=0}^{k-1}\left[\left(s_{1}^{k-1}+s_{2}^{k-1}\right)\right] G_{i} \\
a\left(s_{1}^{k} c_{1}+s_{2}^{k} c_{2}\right)+a^{2} \sum_{i=0}^{k-1}\left[\left(s_{1}^{k-i-1}+s_{2}^{k-i-1}\right)\right] G_{i} \\
s_{1}^{k}\left(s_{1}-a\right) c_{1}+s_{2}^{k}\left(s_{2}-a\right) c_{2}+a \sum_{i=0}^{k-1}\left[\left(\left(s_{1}-a\right) s_{1}^{k-1}+\left(s_{2}-a\right) s_{2}^{k-1}\right)\right] G_{i}
\end{array}\right],
$$

or, equivalently,

$$
\left[\begin{array}{c}
T_{k} \\
C_{k} \\
I_{k}
\end{array}\right]=\left[\begin{array}{l}
s_{1}^{k+1} c_{1}+s_{2}^{k+1} c_{2}+a \sum_{i=0}^{k-1}\left[\left(s_{1}^{k-1}+s_{2}^{k-1}\right)\right] G_{i} \\
a\left(s_{1}^{k} c_{1}+s_{2}^{k} c_{2}\right)+a^{2} \sum_{i=0}^{k-1}\left[\left(s_{1}^{k-i-1}+s_{2}^{k-i-1}\right)\right] G_{i} \\
s_{1}^{k}\left(s_{1}-a\right) c_{1}+s_{2}^{k}\left(s_{2}-a\right) c_{2}+a \sum_{i=0}^{k-1}\left[\left(\left(s_{1}-a\right) s_{1}^{k-1}+\left(s_{2}-a\right) s_{2}^{k-1}\right)\right] G_{i}
\end{array}\right] .
$$

The proof is completed.

The way this method in this theorem reconstructs the Samuelson's model can be also used in models of similar nature. For example, it can be used into other macroeconomic models, or models where the memory effect appears, and models with delays, see (Dassios et al. 2017; Moaaz et al. 2020a, b). In addition, this updated form of Samuelson's model can provide new alternative methods to prove stability of similar dynamical systems, see (Apostolopoulos and Ortega 2018; Boutarfa and Dassios 2017; Dassios 2015b, 2018a). 


\subsection{Initial conditions}

We assume system (4) and the known initial conditions (IC): $Y_{2}$. Note that it is a necessity the initial condition to be $Y_{2}$ because $Y_{k}$ is defined from $T_{k-1}, T_{k-2}$, and for $k=2, T_{2}$ is defined by $T_{1}, T_{0}$.

Definition 4.1 Consider the system (4) with known IC. Then, the IC are called consistent if there exists a solution for the system (4) which satisfies the given conditions.

Proposition 4.1 (See Dassios 2015d) The IC of system (4) are consistent if and only if

$$
Y_{2} \in \text { colspan } Q_{p}+Q D_{2} .
$$

Proposition 4.2 (See Dassios et al. 2014) Consider the system (4) with given IC. Then, the solution for the initial value problem is unique if and only if the IC are consistent. Then, the unique solution is given by the formula

$$
Y_{k}=Q_{p} J_{p}^{k} Z_{2}^{p}+Q D_{k}
$$

where $D_{k}=\left[\begin{array}{c}\sum_{i=0}^{k-1} J_{p}^{k-i-1} P_{1} V_{i} \\ \operatorname{sum}_{i=0}^{q_{*}-1} H_{q}^{i} P_{2} V_{k+i}\end{array}\right]$ and $Z_{2}^{p}$ is the unique solution of the algebraic system $Y_{2}=Q_{p} Z_{2}^{p}+D_{2}$

Proposition 4.2 The singular reformulated Samuelson's model (4) has always a unique solution for any given initial conditions.

Proof The column $Y_{k}$ in (4) is defined as

$$
Y_{k}=\left[\begin{array}{c}
T_{k} \\
C_{k} \\
I_{k}
\end{array}\right]
$$

whereby for $k=2$, we get

$$
Y_{2}=\left[\begin{array}{c}
T_{2} \\
C_{2} \\
I_{2}
\end{array}\right]
$$

or, equivalently, using (2), (3)

$$
Y_{2}=\left[\begin{array}{c}
T_{2} \\
a T_{1} \\
a b\left(T_{1}-T_{0}\right)
\end{array}\right]
$$

or, equivalently,

$$
Y_{2}=\left[\begin{array}{l}
1 \\
0 \\
0
\end{array}\right] T_{2}+\left[\begin{array}{l}
0 \\
1 \\
b
\end{array}\right] a T_{1}+\left[\begin{array}{c}
0 \\
0 \\
-b
\end{array}\right] a T_{0}
$$

But $T_{2}=a(1+b) T_{1}-a b T_{0}+\bar{G}$. Thus 


$$
Y_{2}=\left[\begin{array}{c}
1+b \\
1 \\
b
\end{array}\right] a T_{1}+\left[\begin{array}{c}
-b \\
0 \\
b
\end{array}\right] a T_{0}+\left[\begin{array}{l}
1 \\
0 \\
0
\end{array}\right] \bar{G}
$$

However

$$
\operatorname{colspan} Q_{p}=\left\langle\left[\begin{array}{c}
1+b \\
1 \\
b
\end{array}\right],\left[\begin{array}{c}
-b \\
0 \\
b
\end{array}\right]\right\rangle, \quad Q D_{2}=\left[\begin{array}{l}
1 \\
0 \\
0
\end{array}\right] .
$$

Hence

$$
Y_{2} \in\left\langle\left[\begin{array}{c}
1+b \\
1 \\
b
\end{array}\right],\left[\begin{array}{c}
-b \\
0 \\
b
\end{array}\right]\right\rangle+\left[\begin{array}{l}
1 \\
0 \\
0
\end{array}\right]
$$

or, equivalently,

$$
Y_{2} \in \operatorname{colspan} Q_{p}+Q D_{2} .
$$

Hence from Proposition 4.1, the IC of the singular reformulated Samuelson's model (4) are always consistent and from Proposition 4.1, the singular reformulated Samuelson's model (4) has a unique solution for given IC. The proof is completed.

\section{Conclusions}

In this article, we focused and provided a new alternative formulation of the famous Samuelson macroeconomic model. We proved that this model can be studied via an equivalent singular system of difference equations using pencil theory. We provided properties for existence of solutions. As a future research, it is interesting to study stability and stabilization properties for non-consistent initial conditions. For this case optimization methods, see (Dassios et al. 2015), and concepts from graph theory, see (Cuffe et al. 2016; Dassios et al. 2019), will be required. For this idea, there is already some ongoing research in progress.

Acknowledgements

We would like to express our sincere gratitude to Dr. Sung Cho for his helpful and fruitful discussion that improved this article.

Authors' contributions

All authors contributed equally to the writing of this paper. All authors read and approved the final manuscript.

Funding

Not applicable.

Availability of data and materials

Data sharing is not applicable to this article as no datasets were generated or analyzed during the current study.

Competing Interests

The authors declare that they have no competing interests.

Received: 15 February 2020 Revised: 6 April 2020 Accepted: 11 April 2020

Published online: 24 April 2020 


\section{References}

Apostolopoulos N, Ortega F (2018) The stability of systems of difference equation with non-consistent initial conditions. Dyn Contin Discrete Impuls Syst Series A: Math Anal 25(1):31-40

Apostolopoulos N, Ortega F (2019) On the causality of singular systems of difference equations of first and fractional order. Dyn Contin Discrete Impuls Systems Series A: Math Anal 26:435-445

Barros MF, Ortega F (2019) An optimal equilibrium for a reformulated Samuelson economic discrete time system. J Econ Struct 8:29

Boutarfa B, Dassios I (2017) A stability result for a network of two triple junctions on the plane. Math Methods Appl Sci 40(17):6076-6084

Cuffe P, Dassios I, Keane A (2016) Analytic loss minimization: a proof. IEEE Trans Power Syst 31(4):3322-3323

Dassios I (2012) On non homogeneous linear generalized linear discrete time systems. Circuits Syst Signal Process 31(5):1699-1712

Dassios I (2015a) On a boundary value problem of a singular discrete time system with a singular pencil, dynamics of continuous. Discret Impuls Syst Ser A Math Anal 22(3):211-231

Dassios I (2015b) Stability of basic steady states of networks in bounded domains. Comput Math Appl 70(9):2177-2196

Dassios IK (2015c) Optimal solutions for non-consistent singular linear systems of fractional nabla difference equations. Circuits Syst Signal Process 34(6):1769-1797. https://doi.org/10.1007/s00034-014-9930-2

Dassios I (2015d) Geometric relation between two different types of initial conditions of singular systems of fractional nabla difference equations. Math Methods Appl Sci. https://doi.org/10.1002/mma.3771

Dassios I (2017) Stability and robustness of singular systems of fractional nabla difference equations. Circuits Syst Signal Process 36(1):49-64. https://doi.org/10.1007/s00034-016-0291-x

Dassios I (2018a) Stability of bounded dynamical networks with symmetry. Symmetry 10(4):121

Dassios I (2018b) A practical formula of solutions for a family of linear non-autonomous fractional nabla difference equations. J Comput Appl Math 339:317-328

Dassios I (2019) Analytic loss minimization: theoretical framework of a second order optimization method. Symmetry 11(2):136

Dassios IK, Baleanu D (2013) On a singular system of fractional nabla difference equations with boundary conditions. Bound Value Probl 2013:148

Dassios IK, Baleanu DI (2015) Duality of singular linear systems of fractional nabla difference equations. Appl Math Model 39(14):4180-4195. https://doi.org/10.1016/j.apm.2014.12.039

Dassios I, Baleanu D (2018) Caputo and related fractional derivatives in singular systems. Appl Math Comput 337:591-606

Dassios I, Devine M (2016) A macroeconomic mathematical model for the national income of a union of countries with interaction and trade. J Econ Struct 5:18

Dassios IK, Kalogeropoulos G (2013) On a non-homogeneous singular linear discrete time system with a singular matrix pencil. Circuits Syst Signal Process 32(4):1615-1635

Dassios I, Kalogeropoulos G (2014) On the stability of equilibrium for a reformulated foreign trade model of three countries. J Ind Eng Int 10(3):71

Dassios IK, Szajowski K (2016) Bayesian optimal control for a non-autonomous stochastic discrete time system. Appl Math Comput 274:556-564

Dassios I, Zimbidis A (2014) The classical Samuelson's model in a multi-country context under a delayed framework with interaction. Dyn Contin Discret Impuls Syst Ser B Appl Algorithms 21(4-5b):261-274

Dassios I, Zimbidis A, Kontzalis C (2014a) The delay effect in a stochastic multiplier-accelerator model. J Econ Struct 3:7

Dassios I, Baleanu D, Kalogeropoulos G (2014b) On non-homogeneous singular systems of fractional nabla difference equations. Appl Math Comput 227:112-131

Dassios I, Fountoulakis K, Gondzio J (2015) A preconditioner for a primal-dual Newton conjugate gradients method for compressed sensing problems. SIAM J Sci Comput 37:A2783-A2812

Dassios I, Jivkov AP, Abu-Muharib A, James P (2017) A mathematical model for plasticity and damage: a discrete calculus formulation. J Comput Appl Math 312:27-38

Dassios I, Tzounas G, Milano F (2019) The Mobius transform effect in singular systems of differential equations. Appl Math Comput 361:338-353

Dassios I, Cuffe P, Keane A (2019) Calculating nodal voltages using the admittance matrix spectrum of an electrical network. Mathematics $7(1): 106$

Dassios I, Tzounas G, Milano F (2020) Participation factors for singular systems of differential equations circuits. Syst Signal Process 39(1):83-110

Dassios I, Baleanu D (2018) Optimal solutions for singular linear systems of Caputo fractional differential equations. Math Methods Appl Sci

Dorf RC (1983) Modern control systems, 3rd edn. Addison-Wesley, Boston

Klamka J (2010) Controllability and minimum energy control problem of fractional discrete-time systems. New trends in nanotechnology and fractional calculus. Springer, New York, pp 503-509

Klamka J, Wyrwał J (2008) Controllability of second-order infinite-dimensional systems. Syst Control Lett 57(5):386-391

Kuo BC, (1996) Automatic control systems, 5th edn. Prentice Hall, Upper Saddle River

Lewis FL (1987) Recent work in singular systems. In: Proc Int Symp singular systems, Atlanta, GA, pp 20-24

Lewis FL (1986) A survey of linear singular systems. Circuits Syst Signal Process 5:3-36

Lewis FL (1992) A review of 2D implicit systems. Automatica 28(2):345-354

Liu M, Dassios I, Milano F (2019a) On the stability analysis of systems of neutral delay differential equations. Circuits Syst Signal Process 38(4):1639-1653

Liu M, Dassios I, Tzounas G, Milano F (2019b) Stability analysis of power systems with inclusion of realistic-modeling of WAMS delays. IEEE Trans Power Syst 34(1):627-636

Liu M, Dassios I, Tzounas G, Milano F (2020) Model-independent derivative control delay compensation methods for power systems. Energies 13(2):342 
Liu M, Dassios I, Milano F (2017) Small-signal stability analysis of neutral delay differential equations. IECON 2017—43rd annual conference of the IEEE industrial electronics society. IEEE, New York, pp 5644-5649

Milano F, Dassios I (2016) Small-signal stability analysis for non-index 1 Hessenberg form systems of delay differentialalgebraic equations. IEEE Trans Circuits Syst I Regul Pap 63(9):1521-1530

Milano F, Dassios I (2017) Primal and dual generalized eigenvalue problems for power systems small-signal stability analysis. IEEE Trans Power Syst 32(6):4626-4635

Moaaz O, Dassios I, Bazighifan O, Muhib A (2020) Oscillation theorems for nonlinear differential equations of fourth-order. Mathematics 8(4):520

Moaaz O, Dassios I, Bazighifan O (2020) Oscillation criteria of higher-order neutral differential equations with several deviating arguments. Mathematics 8(3):402

Ogata K (1987) Discrete time control systems. Prentice Hall, Upper Saddle River

Ortega F, Apostolopoulos N (2018) A generalised linear system of difference equations with infinite many solutions. Dyn Contin Discret Impuls Syst Ser B Appl Algorithms 25:397-407

Podlubny I (1999) Fractional differential equations, mathematics in science and engineering. Academic Press, San Diego pxxiv +340

Puu T, Gardini L, Sushko I (2004) A Hicksian multiplier-accelerator model with floor determined by capital stock. J Econ Behav Organ 56:331-348

Rosser JB (2000) From catastrophe to chaos: a general theory of economic discontinuities. Academic Publishers, Boston

Samuelson P (1939) Interactions between the multiplier analysis and the principle of acceleration. Rev Econ Stat 21:75-78

Tzounas G, Dassios I, Milano F (2020) Modal participation factors of algebraic variables. IEEE Trans Power Syst 35(1):742-750

\section{Publisher's Note}

Springer Nature remains neutral with regard to jurisdictional claims in published maps and institutional affiliations.

\section{Submit your manuscript to a SpringerOpen ${ }^{\circ}$ journal and benefit from:}

- Convenient online submission

- Rigorous peer review

- Open access: articles freely available online

- High visibility within the field

Retaining the copyright to your article

Submit your next manuscript at $\gg$ springeropen.com 